\title{
Study on the Expression of Organic Anion Transporting Polypeptide (oatp2a1) in Rat with Spleen Deficiency Syndrome and the Exploration of Clinical Significance
}

\author{
Ai-Zhen Pan ${ }^{1}$, Yao-Kun Hao ${ }^{1}$, You-Wu Lin ${ }^{1}$, Ze-Xiong Chen ${ }^{1}$, Shi-Jun Zhang ${ }^{1 \#,}$ \\ Xiao Dong', Bin $\mathrm{Hu}^{2}$ \\ ${ }^{1}$ Department of Traditional Chinese Medicine, The First Affiliated Hospital of Sun Yat-Sen Universty, \\ Guangzhou, China \\ ${ }^{2}$ DaAn Gene Co., Ltd. of Sun Yat-Sen University, Guangzhou, China \\ E-mail: " ${ }$ hsjun1967@yahoo.com.cn \\ Received June 16, 2011; revised June 28, 2011; accepted July 1, 2011
}

\begin{abstract}
Objective: to explore the mechanism of transportation and transformation of dampness by the way of the expression of organic anion transporting polypeptide (oatp) superfamily member 2a1 (oatp2a1) mRNA in rat with spleen deficiency syndrome and the significance in transportation and transformation of dampness. Methods: 32 wistar male rats were divided randomly into four groups: normal group $(n=6)$, normal + AA group $(n=6)$, spleen deficiency group $(n=10)$, Spleen deficiency + AA group $(n=10)$. After reserpine-induced spleen deficiency model was made, intragastric administration of aristolochic acid (AA) was adopted for three days, the expression of oatp2a1 mRNA were detected in the tissues of lung, liver, kidney, stomach, small intestine and large intestine in four groups by using Fluorescent Quantitative-Polymerase Chain Reaction (FQ-PCR). Results: the expression of oatp2a1 mRNA in above six tissues could be detected. The expression of oatp2a1 mRNA in liver tissue of rat with spleen deficiency syndrome was up-regulated compared to normal group $(P=0.035, P<0.05)$, the expression of oatp2al mRNA in small intestinal tissue of rat with spleen deficiency syndrome was down-regulated compared to normal group $(P=0.004, P<0.01)$, the expression of oatp2a1 in intestinal tissue in normal + AA group is down-regulated compared to normal group $(P=0.032, P<0.05)$. Conclusions: oatp2a1 might be one of the material basis involved in transportation and transformation of dampness. The changes of expression of oatp2a1 mRNA in small intestine, liver tissue suggests that small intestine, liver might play an important role in the transportation and transformation of dampness in the state of spleen deficiency. We further concluded that the function of spleen's governing transportation and transformation of dampness was not only including the function of the gastrointestinal, but also part of the liver function in some degree, which needs to be further studied.
\end{abstract}

Keywords: Dampness, Spleen Deficiency Syndrome, oatp2a1

\section{Introduction}

Metabolism of body fluid includes the transportation and transformation of water, essence of water and grain, and dampness, the generation, distribution and excretion of water involves coordination of various visceral functions.

*Source of support: this work was supported by the Major Special Program of the "Eleventh Five-year Plan" for Sci \& Tech Research of China (No. 2009ZX10005-016), National Natural Science Foundation of China (No. 81072806) and Natural Science Foundation of Guangdong Province (No. 9151008901000117). especially the lung, spleen (stomach), kidney, and triple energizer. In Jing Mai Bie Lu of Su Wen(Plain Questions), it is said that water enters the stomach, goes upward to the spleen, which can distribute essence upwards to the lung, which can regulate water passage to transport water downwards to the bladder, through which water and essence are distributed to the whole body. Currently, more and more research has been carried out about transportation and transformation of water as well as essence of water and grain, but the research about damp- 
ness mechanism is relative few, which holds back the development of dampness metabolism theory. Many researchers studied water liquid metabolism by the way of Aquaporin and antidiuretic hormone (ADH), few of previous studies were carried out about the mechanism of the dampness by the way of oatps.

A number of organic anion transporters (human OATP/ SLCO, rodent (oatp/Slco) can efficiently transport a variety of foreign organisms such as drugs, xenobiotics, and endogenous substances so on, oatp2al is expressed widely in human and rat tissues. During recent years, it has become increasingly recognized that oatps play important roles in drug absorption and disposition and are critically involved in the cellular uptake of drugs in tissues important for pharmacokinetics, such as intestine, liver and kidney. Although several members of multi specific oatps that mediate the elimination of organic anions in the liver and other organs have been identified. few studies have been carried to elucidate the role of oatp2a1 in elimination of AA or dampness. So our objective of study is to explore the mechanism of transportation and transformation of dampness by the way of the expression of oatp2a1 in rat with spleen deficiency syndrome and the significance in transportation and transformation of dampness. The findings of the report are as follows.

\section{Methods}

\subsection{Animals}

32 six-week-old wistar male rats weighting to 150 - 180 $\mathrm{g}$ were purchased from the animals central of Guangdong province, animals were housed in a temperature-, light-, and humidity-controlled environment. License number is SCXK2008-0002, Guangdong License number is 2008A 020 .

\subsection{Drugs and Reagents}

Aristolochic acid (AA) was obtained from YI-ER-TA Pharmaceutical Limited Company of Wu Huo, the purity of AA was 99\%, $20 \mathrm{mg} / \mathrm{PC}$. Reserpine injection was purchased from BANG-MING Pharmaceutical Limited Company of guang dong, $1 \mathrm{mg} / \mathrm{pc}$. RNAiso ${ }^{\mathrm{TM}}$ Plus isolation kit was purchased from TaKaRa (D9108A) $5 \times$ RT buffer, $5 \times$ quantitative PCR buffer, dNTPs, MMLV, Taq enzyme was purchased from Guangzhou Huayin Medical Technology Co., Ltd., OligoT was purchased from Promega. Conventional sub-analytically pure chemical reagents.

\subsection{Instruments}

Sigma desktop high-speed refrigerated centrifuge machines, MJ gradient PCR instrument, MJ Opticon2 automatic quantitative PCR instrument, sanyo ultra low temperature refrigerator.

\subsection{Animal Group}

After being purchased, the rats were treated with free diet, activity, sleep until weighting to 250 - $280 \mathrm{~g}$ during the first two weeks. The rats were randomly divided into 4 groups: normal group $(n=6)$, normal group + AA group $(n=6)$, spleen deficiency group $(n=10)$, spleen deficiency + AA group $(n=10)$.

\subsection{Production of Spleen Deficiency Rat Model}

10 rats were given reserpine $5 \mathrm{mg} / \mathrm{kg} / \mathrm{d}$, subcutaneous injection for continuous 15 days with normal feeding, which references and improves the spleen deficiency rat model documentation [1]. The criterion of spleen defi- ciency are as follows: 1) listlessness, somnolence; 2) anorexia; 3) loose stool (anal dirty); 4) abatement of drinking water.

\subsection{Group Intervention Methods}

After reserpine-induced spleen deficiency model was made, intragastric administration of aristolochic acid (20 $\mathrm{mg} / \mathrm{kg} / \mathrm{d}$ ) was adopted to the normal + AA group and spleen deficiency + AA group of rats, continuously intragastric administration of AA for 3 days [2]. The normal group was intragastricly administrated by $0.9 \%$ saline $(10 \mathrm{ml} / \mathrm{kg})$ for 3 days. Each group of rats in the last 6 hours after administration were sampled.

\subsection{Observation of General Symptoms}

General symptoms were closely observed every day of experiment, such as the quantity of diet and drinking water, body weight, growth condition, excrement situation, the state of mind and activity, complexion and so on.

\subsection{Specimen Collection}

The rats were anesthetized with $3 \%$ pentobarbital sodium by the way of intraperitoneal injection $0.2 \mathrm{ml} / 100 \mathrm{~g}$, each piece of tissue was drew from lung, liver, kidney, stomach, small intestine, large intestine, each size of sample was about $5 \mathrm{~mm} * 5 \mathrm{~mm}$, putted into the freezing tube; all samples were preserved within 15 minutes into the $-80^{\circ} \mathrm{C}$ refrigerator. 


\section{9. oatp2a1 Gene Expression}

Total RNA extraction: total RNA extraction used different hydroxyl guanidine chloride-chloroform method, to test $\mathrm{OD}$ values.

Synthesis of primers: to find the target gene mRNA sequence in GenBank, to design specific primers in the CDS area, probes used for primer design were software Primer express 2.0 software. The specimens were sent to the British Biotechnology Co.Ltd. Shanghai for synthesis, desktop equipment for the ABI 3900 High-throughput DNA synthesizer. Protein gene sequences: upstream 5'-tccgaaacaggtacctgggcc-3', downstream 5'-tccagctgatgaagaagagcagc-3', fluorescent group sequence: 5'-FAMcagatggtctacaaggccttgggcacac-BHQ1-3', reference protein $\beta$-acting gene sequences were as follows: upstream 5'-gaagctcatttcctggtatgacaatg-3', downstream 5'-cttggaggccatgtaggccatg-3', fluorescent group gene sequence: 5'FAM-tatggctacagcaacagggtggtggac-BHQ1-3'.

Reverse transcriptase polymerase chain reaction (RTPCR) $10 \mu \mathrm{l}$ RNA template reverse transcription reaction, apparatus for the MJ gradient PCR instrument (United States Bio-Rad Company), the reaction system as follows: $5 \times$ Buffer $4 \mu \mathrm{L}$, dNTP $1 \mu \mathrm{L}$, RNase Free water, 2 $\mu \mathrm{l}$, Oligo dT18 $1 \mu \mathrm{l}$, MMLV enzyme $2 \mu \mathrm{l}$.

\subsection{Quantitative PCR Reactions}

Positive standard and its gradient pre-trial preparation positive product of pre-trial preparation was electrophored by $2 \%$ low melting agarose gel after PCR amplification (containing ethidium bromide, prepared with TAE buffer), target band was cut in the long-wave UV, Determination of OD $260 / 280>1.8$, which show the purity is up to grade. Measured with OD260 values and fragment length data conversion from the concentration (copy/ $\mu \mathrm{l}$ ), as the positive standard. Take positive standard $5 \mu$ diluted by 10 times $(45 \mu \mathrm{l}$ water and adequate mixing), followed by dilution continues, the positive quantitative standard was prepared gradient.

positive standard samples under test and the following reaction system $(25 \mu \mathrm{l}$ system): Slco2al and gapdh follow the same system of 10X PCR Buffer $2.5 \mu \mathrm{l}, 2.5 \mathrm{mM}$ dNTP $2 \mu \mathrm{l}, \mathrm{H}_{2} \mathrm{O} 16 \mu \mathrm{l}, 15 \mathrm{pmol} / \mu \mathrm{l}$ primer $1+1 \mu \mathrm{l}, 10$ $\mathrm{pmol} / \mu \mathrm{l}$ probe, $0.3 \mu \mathrm{l}, 5 \mathrm{U} / \mu \mathrm{l}$ Taq $0.2 \mu \mathrm{l}$, Template $2 \mu \mathrm{l}$. $94^{\circ} \mathrm{C} 2 \mathrm{~min} ; 94^{\circ} \mathrm{C} 5 \mathrm{sec}, 60^{\circ} \mathrm{C}, 30 \mathrm{sec}$, reading FAM fluorescence values, 40 cycle; $30^{\circ} \mathrm{C} 5 \mathrm{sec}$.

\subsection{Statistical Analysis}

Quantitative Instrument aMJ Opticon2 automatic quantitative PCR instrument (United States Bio-Rad company). After reaction, the computer automatically analyzed and calculated results. The results were shown by the table under the Copies value (i.e. copy number) analysis, with $\mathrm{B}$, i.e. $\mathrm{B}=$ copy number $/ \mu \mathrm{l} \mathrm{cDNA}$. Taking into account the total RNA concentration of each sample differences, the final results are translated by the following formula: $\mathrm{A}=\mathrm{B}$ (target gene) $\div \mathrm{B}$ (internal reference gene). A statistical value is required when the final value.

Application of statistical methods statistical software SPSS 13.0 for windows, using analysis of variance (ANOVA, homogeneity of variance) on the results of statistical analysis.

\section{Results}

\subsection{Comparison of General Symptoms}

After reserpine-induced spleen deficiency model was made, the rats had those symptoms such as loss of appetite, listlessness, somnolence, anorexia, Loose stool (anal dirty), thin, swallow complexion, and so on. There was significant difference between the normal group and the spleen deficiency syndrome group.

\subsection{Expression of oatp2a1 mRNA}

The expression of oatp2a1 mRNA was detected in the tissues of lung, liver, kidney, stomach, small intestinal and large intestine in four groups by using Fluorescent Quantitative-Polymerase Chain Reaction (FQ-PCR).

Oatp2a1 mRNA was expressed in lung, liver, kidney, stomach, small intestine and large intestine tissues in normal group, oatp2a1 mRNA expression analysis of variance had statistic significant $(P=0, P<0.01)$; Pairwise comparison results were as follows: the expression of oatp2a1 mRNA in lung tissue was higher than that in liver tissue $(P=0.03, P<0.05)$, the expression of oatp2al mRNA in lung tissue was lower than that in kidney tissue $(P=0, P<0.01)$, the expression of oatp2a1 mRNA in lung was lower than that in gastric tissues $(P=$ $0.03, P<0.05)$, the expression of oatp2a1 mRNA in lung tissue is lower than that in small intestine $(P=0, P<$ 0.01 ), the expression of oatp2a1 mRNA in liver tissue was lower than that in kidney tissue $(P=0, P<0.01)$, the expression of oatp2a1 mRNA in liver tissue was lower than that in small intestinal tissues $(P=0.006, P<$ 0.01 ), the expression of oatp2a1 mRNA in stomach tissue was lower than that in kidney tissue $(P=0, P<0.01)$, the expression of oatp2a1 mRNA in large intestine tissue was lower than that in the kidney $(P=0, P<0.01)$, the expression of oatp2al mRNA in stomach tissues was lower than that in small intestinal tissues $(P=0.006, P<$ $0.01)$, the expression of oatp2a1 mRNA in large intestine tissues was lower than that of small intestinal tissues $(P$ 
$=0, P<0.01)$. In summary, the oatp2a1 mRNA expression was the highest in kidney and small intestine tissue of 6 groups, followed by the liver, stomach, large intestine, the expression of oatp2a1 mRNA is least in lung tissue. While, there was no significant difference between the kidney and small intestine tissues. There was no statistic- cal significance among the three tissues of liver, stomach, and large intestine.

The expression of oatp2a1 mRNA in liver tissue of rat with spleen deficiency syndrome was up-regulated compared to normal group $(P=0.035, P<0.05)$, the expression of oatp2al mRNA in small intestinal tissue of rat with spleen deficiency syndrome was down-regulated compared to normal group $(P=0.004, P<0.01)$, the expression of oatp2a1 mRNA in intestinal tissue in normal + AA group is down-regulated compared to normal group $(P=0.032, P<0.05)$. The results were shown in Table 1.

\section{Discussion}

Water is the main component of the human body, in any individual, the body water content stays remarkably constant from day to day. Water as solute liquid carrier, its contents includes essence of water and grain, water, dampness (food metabolites, drug metabolites, endogenous and exogenous toxins, etc.) [4-6].The generation, distribution and excretion of water is a complicated physiological process involving functions of various viscera, especially the lung, spleen, and kidneys, and spleen's function is the most important.

In present studies of water metabolism concerning lung, spleen and kidney. Li Shuweng, etc., studied water liquid metabolism from the Aquaporin-2 (AQP2) and antidiuretic hormone (ADH) approach of the lung dysfunction, Li-Qun, Aquaporin 1 (AQP1) explored water metabolism on the basis of rat models of the kidney Yang and Qi deficiency [7,8]. Lao Shaoxian etc., used water Aquaporin as a point index to carry out the study of water liquid metabolism of splenic heat and humidity syndrome type, they found spleen's governing transportation and transformation function is closely related to water AQP, the disorder of water AQP-mediated transportation can lead to splenic dysfunction of transportation and transformation water liquid. Internal water retention resulted in a range of water fluid pathological changes such as absorption, distribution, metabolism disorder [9-11]. These studies mainly concentrated on the water and the essence of water and grain metabolism, however, few of previous studies were carried out about the mechanism of the dampness.

Body produces a large number of dampness including food, metabolites, endogenous and exogenous toxins, drug metabolites, etc. and foreign organisms in metabolism. Current research shows that oatps can efficiently transfer a variety of foreign organisms and play a key role in transit dampness $[12,13]$. Members of the organic anion transporter family functio $\mathrm{n}$ as uptake transporters of a wide variety of drugs, xenobiotics and endogenous substances. In humans and mice, 11 and 15 family members, respectively, have thus far been identified. Previous studies have considered only transport of organic anions oatp, but recent research confirms oatp has a wide range of substrates, including cationic, anionic, gender and anion complex, if oatps dysfunction, will cause dampness of the absorption, distribution and excretion of obstacles, a partial or whole body fluid balance disorders, leading to diseases [14-16].

The study found that oatp2a1 mRNA was expressed in the lung, liver, kidney, stomach, small intestine and large intestine tissues in the spleen deficiency model group and normal group, suggested that oatp2a1 mRNA expression might be one of the foundation of the three viscerals (lung, spleen and kidney) as center in the coregulation of dampness. The expression of oatp2a1 mRNA in liver tissue of rat with spleen deficiency syndrome was up-regulated compared to normal group, the expression of oatp2a1 mRNA in small intestinal tissue of

Table 1. oatp2a1mRNA expression levels in 6 tissues of each group $(\bar{x} \pm s)$.

\begin{tabular}{|c|c|c|c|c|}
\hline tissue & $\operatorname{normal}(n=6)$ & normal $+\mathrm{AA}(n=6)$ & reserpine $(n=10)$ & reserpine $+\mathrm{AA}(n=10)$ \\
\hline lung & $1.49 \mathrm{E}-02 \pm 1.22 \mathrm{E}-03$ & $2.08 \mathrm{E}-02 \pm 4.18 \mathrm{E}-03$ & $1.80 \mathrm{E}-02 \pm 6.62 \mathrm{E}-03$ & $2.07 \mathrm{E}-02 \pm 5.74 \mathrm{E}-03$ \\
\hline liver & $2.34 \mathrm{E}-02 \pm 2.56 \mathrm{E}-03$ & $2.07 \mathrm{E}-02 \pm 5.35 \mathrm{E}-03$ & $3.15 \mathrm{E}-02 \pm 1.10 \mathrm{E}-02 *$ & $3.42 \mathrm{E}-02 \pm 3.58 \mathrm{E}-03$ \\
\hline kidney & $3.88 \mathrm{E}-02 \pm 8.24 \mathrm{E}-03$ & $3.83 \mathrm{E}-02 \pm 1.61 \mathrm{E}-02$ & $3.92 \mathrm{E}-02 \pm 1.09 \mathrm{E}-02$ & $3.86 \mathrm{E}-02 \pm 9.00 \mathrm{E}-03$ \\
\hline stomach & $2.33 \mathrm{E}-02 \pm 8.50 \mathrm{E}-03$ & $3.08 \mathrm{E}-02 \pm 8.67 \mathrm{E}-03$ & $2.35 \mathrm{E}-02 \pm 9.10 \mathrm{E}-03$ & $2.90 \mathrm{E}-02 \pm 1.04 \mathrm{E}-02$ \\
\hline small intestine & $3.47 \mathrm{E}-02 \pm 9.58 \mathrm{E}-03$ & $2.33 \mathrm{E}-02 \pm 8.49 \mathrm{E}-03 \boldsymbol{\Delta}$ & $2.04 \mathrm{E}-02 \pm 6.23 \mathrm{E}-03 \triangle \triangle$ & $2.78 \mathrm{E}-02 \pm 1.03 \mathrm{E}-02$ \\
\hline large intestine & $1.65 \mathrm{E}-02 \pm 4.37 \mathrm{E}-03$ & $1.83 \mathrm{E}-02 \pm 5.91 \mathrm{E}-03$ & $2.05 \mathrm{E}-02 \pm 1.01 \mathrm{E}-02$ & $2.32 \mathrm{E}-02 \pm 1.11 \mathrm{E}-02$ \\
\hline
\end{tabular}

Note: compared with normal group, $* P<0.05, * * P<0.01$; compared with the normal group, $\triangle P<0.05, \triangle \triangle P<0.01$. Compared with normal group, $\Delta P$ $<0.05, \Delta \Delta P<0.01$. 
rat with spleen deficiency syndrome was down-regulated compared to normal group, the expression of oatp2a1 mRNA in intestinal tissue in normal + AA group was down-regulated compared to normal group, while there was no difference in the other four kinds of tissues between two groups. According to Chinese medicine theory, the failure of spleen qi in the transportation and transformation water fluids results in retention of dampness, retenting in the gastrointestinal system, obstructing in the meridians, or remaining in the organs. As a bidirectional transfer protein [17], oatp2al mRNA was downregulated in the small intestine tissue of rat with spleen deficiency syndrome, which means a self-protective reaction of the body to reduce the absorption of dampness in small intestine. In the same time, it was interesting to find that the rats with spleen deficiency syndrome had loose stool, which might indicate that the body evacuated dampness by loose stool. oatp2a1 mRNA expression in liver tissue of rat with spleen deficiency syndrome was higher than that of normal rat, which suggested the enhanced ability in transporting dampness. This indicates that the liver and small intestine play an important role in the transportation and transformation of dampness, furthermore, it reminds us that the function of spleen's governing transportation and transformation of dampness was not only including the functions of the gastrointestinal, but also part of the liver function in some degree.

After intragastric administration of aristolochic acid, oatp2a1 mRNA expression in mall intestine tissue of rat in the normal + AA group model rats was significantly down-regulated compared to the normal group. As we all known, the small intestine is the main absorbing organ. The decreased expression of oatp2a1 mRNA in the intestinal tissue when stimulated by external organic anions indicated that the small intestine reduced the absorption of dampness, it might be the body self-protective response to exogenous dampness.

Under exogenous dampness stimulation, the expression of oatp2a1 mRNA showed no significant reduction or elevation, which might suggest that the exogenous dampness makes an important difference in oatp2al mRNA expression, while this was not obvious in state of spleen deficiency syndrome. This is perhaps a response of body to transporting and transforming dampness.

In summary, oatp2a1 might be one of material basis involved in transportation and transformation of dampness, the changes of oatp $2 \mathrm{a} 1 \mathrm{mRNA}$ expression in small intestine and liver tissue suggested that small intestine and liver might play an important role in the transportation and transformation of dampness in the state of spleen deficiency. We further concluded that the function of spleen's governing transportation and transformation of dampness was not only related to the functions of the gastrointestinal, but also to the function of the liver in some degree, which needs further study.

\section{Acknowledgements}

The authors would like to thank DA-AN GENE company for assistance with gene testing and we also thank Feng WANG professor, director of central research laboratories. YI-ER-TA Pharmaceutical Limited Company of wuhuo for providing drugs and reagents. This work was supported by the major special program of the "Eleventh Five-Year Plan" for Sci \& Tech Research of China, the National Natural Science Foundation of China and the Natural Science Foundation of Guangdong province.

\section{References}

[1] Y. K. Li, "Methodology of Chinese herbs pharmacology," Shanghai Science and Technology Publishing Company, Shanghai, 2006, pp. 162-163.

[2] H. Li, Z. H. Lui, J. P. Wang and H. P. Cheng, "Aristolochic Acid-I Induces Acute Renal Failure in Rats," Chinese Journal of Nephrology, Vol. 18, No. 1, 2002, pp. 53-55.

[3] S. W. Li and Q. H. Wu, "Study on Expression of CYP11B2mRNA in Kidney Tissue in Rat Models of Kidney-Yang-Deficiency Syndrome Induced by Adenine," China Journal of Experimental Traditional Medical Formulae, Vol. 16, No. 2, 2010, pp. 64-67.

[4] H. R. Mortaheb, H. Kosuge, et al., "Study on Removal of Cadmium from Wastewater by Emulsion Liquid Membrane," Journal of Hazardous Materials, Vol. 165, No. $1-3,2009$, pp. 630-636. doi:10.1016/j.jhazmat.2008.10.039

[5] N. Li and H. K. Lee, "Assessment of Colloid Formation and Physical State Distribution of Trace Polycyclic Aromatic Hydrocarbons in Aqueous Samples," Analytical Chemistry, Vol. 73, No. 21, 2001, pp. 5201-5206. doi:10.1021/ac010570x

[6] T. S. Wiedmann and A. Ravichandran, "Ultrasonic Nebulization System for Respiratory Drug Delivery," Pharmaceutical Development and Technology, Vol. 6, No. 1, 2001, pp. 83-89. doi:10.1081/PDT-100000016

[7] S. W. Li and Q. W. Wu, "Study on Expression of CYP11B2mRNA in Rat Models of Kidney-Yang-Deficiency Syndrome Tissue Induced by Adenine," China Journal of Experimental Traditional Medical Formulae, Vol. 16, No. 2, 2010, pp. 64-67.

[8] Y. Li, L. Q. He, X. Zhong, J. Yi and X. B. He, "Change of Aquaporin-1 in Rat Models of Kidney-Yang-Deficiency Syndrome," Journal of Chinese Integrative Medicine, Vol. 6, No. 5, 2008, pp. 498-501. doi: $10.3736 /$ jcim 20080513

[9] W. X. Mei, S. X. Lao and Z. Zhong "Relationship between Gene Expressions of Aquaporin 3 and 4 and Vari- 
ous Degrees of Spleen-Stomach Dampness-Heat Syndrome in Chronic Superficial Gastritis," Journal of Chinese Digestion, Vol. 8, No. 2, 2010, pp. 111-115.

[10] W. X. Mei and S. X. Lao, "Study on Correlation between Aquaporin 3, 4 Gene Expression in Gastric Mucosa and Severity of Pi-Wei Damp-Heat Syndrome in Patients with Chronic Superficial Gastritis," Chinese Journal of Integrative Medicine, Vol. 27, No. 10, 2007, pp. 891-893.

[11] G. X. Chen, S. X. Lao and Z. X. Huang, "Effect of Chinese Herbs on Expression of Aquaporin 3,4 Gene in Gastric Mucosa of Patients with Pi-Wei Damp-Heat SyndroMe," Chinese Journal of Integrative Medicine, Vol. 25, No. 3, 2005, pp. 199-202.

[12] Z. M. Zair, J. J. Eloranta and B. Stieger and G. A. KullakUblick, "Pharmacokinetics of OATP (SLC21/SLCO), OAT and OCT (SLC22) and PEPY (SLC15) Transporters in the Intestine, Liver and Kidney," Pharmacogenomics, Vol. 9, No. 5, 2008, pp. 597-624. doi:10.2217/14622416.9.5.597

[13] F. S. Sarr, C. Andréand and Y. C. Guillaume, "A Novel Biochromato Graphic oatp2 Column to Study the Transmembrane Transport of Statins," Journal of Pharmaceutical and Biomedical Analysis, Vol. 52, No. 1, 2010, pp.
93-98. doi:10.1016/j.jpba.2009.11.029

[14] B. Hagenbuch and C. Gui, "Xenobiotic Transporters of the Human Organic Anion Transporting Polypeptides (OATP) Family," Xenobiotica, Vol. 38, No. 7-8, 2008, pp. 778-801. doi:10.1080/00498250801986951

[15] J. König, A. Seithel, U. Gradhand and M. F. Fromm, "Pharmacogenomics of Human OATP Transporters," Naunyn-Schmiedeberg's Archives of Pharmacology, Vol. 372, No. 6, 2006, pp. 432-443.

[16] K. A. Wojtal, J. J. Eloranta, P. Hruz, H. Gutmann, J. Drewe, A. Staumann, C. Beglinger, M. Fried, G. A. Kullak-Ublick and S. R. Vavricka, "Changes in mRNA Expression Levels of Solute Carrier Transporters in Inflammatory Bowel Disease Patients," Drug Metabolism and Disposition, Vol. 37, No. 9, 2009, pp. 1871-1877. doi:10.1124/dmd.109.027367

[17] B. Hagenbuch and P. J. Meier, "Organic Anion Transporting Polypeptides of the OATPPSLC21 Family: Phylogenetic Classificationas OATP/SLCO Superfamily, New Nomenclature and Molecular/Functional Properties," Pflugers Arch, Vol. 447, No. 5, 2004, pp. 653-665. 